\title{
Gut morphology of young pigs fed diets differing in standardized ileal digestible threonine and wheat gluten used as a source of non-essential amino acids
}

\author{
E. Święchํㅜ, M. Barszcz, A. Tuśnio and M. Taciak \\ The Kielanowski Institute of Animal Physiology and Nutrition, Polish Academy of Sciences \\ Instytucka 3, 05-110 Jabłonna, Poland
}

KEY WORDS: pigs, threonine, wheat gluten, gut morphology

Received: 8 December 2015

Revised: 27 June 2016

Accepted: 26 August 2016

${ }^{1}$ Corresponding author: e-mail: e.swiech@ifzz.pl

\begin{abstract}
The aim of the study was to determine whether non-essential amino acids (NEAA) in form of wheat gluten (WG) have a threonine (Thr)sparing effect on gut structure in young pigs. A two-factorial experiment was conducted on 12 groups of 6 pigs. Pigs of $12.5 \mathrm{~kg}$ initial body weight (BW) were fed for about 20 days diets differing in the Thr level (5.1, 5.7, 6.3 and $6.9 \mathrm{~g}$ standardized ileal digestible $\left.\mathrm{Thr} \cdot \mathrm{kg}^{-1}\right)$ and WG level $(20.4,40.4$ and $60.4 \mathrm{~g}$ of WG protein $\cdot \mathrm{kg}^{-1}$ of respective WG20, WG40 and WG60 diets). After slaughter at about $22.4 \mathrm{~kg}$ of BW, samples of duodenum, middle jejunum, ileum and proximal colon were taken for histological analysis. Morphology of all segments was not affected neither by Thr level nor by Thr and WG levels interaction, whereas effect of WG level differed among segments. In the duodenum, villi length and tunica mucosa thickness were decreased by WG60. In the duodenum and proximal colon, crypt depth was greater in WG20 than WG60 and/or WG40 groups, whereas in the ileum was greater in WG60 than WG20 group, and in the middle jejunum it was not affected. Villi length : crypt depth ratio was affected by WG only in the ileum, being greater in WG20 group. Myenteron was thicker in the ileum and proximal colon in WG60 than WG20 and WG40 group, respectively. The results do not evidence a sparing effect of NEAA provided by WG on Thr utilization for maintaining the proper gut structure in young pigs.
\end{abstract}

\section{Introduction}

The gut is a highly differentiated and complex organ responsible for digestion, absorption and metabolism of nutrients including protein and amino acids (AA). The maintenance of normal gastrointestinal functions is crucial for animal growth, development and health. Diet composition is an important factor influencing the structure of the small and large intestine. Changes in the gut structure, as villus atrophy and crypt hyperplasia, are generally associated with reduction in enzyme activity and decrease of digestive and absorptive capacity of the gut (Pluske et al., 1997). Villus and crypt morphology, therefore, is often used for describing intestinal functions. Reduction of villus length indicates enterocytes damage, whereas crypt depth is related to cell proliferation and enterocytes maturity (Cera et al., 1988; Dunsford et al., 1989). Villi length : crypt depth ratio is a useful criterion of digestive and absorptive capacity of the small intestine, and myenteron thickness is associated with digesta passage rate and 
nutrient digestibility (Pluske et al., 1997; Montagne et al., 2003). The duodenum is the first segment of the small intestine, which is mainly responsible for enzyme secretion, whereas the jejunum and ileum for absorption processes.

Threonine (Thr) is an essential AA, which is involved in such physiological functions like digestion (Burrin et al., 2001) and immunity (Wang et al., 2006). Significant part of the dietary Thr is used by the intestinal tissue (Bertolo et al., 1998; Stoll et al., 1998), and deficiency or excess of dietary Thr may reduce the synthesis of protein in intestinal mucosa (Wang et al., 2007). Thr is particularly important for the synthesis of mucins and constitutes $30 \%$ of mucin AA. Maintenance requirement for $\mathrm{Thr}$ depends on secretion, reutilization and loss of mucins, and lower availability of this AA may reduce the mucin production and impair barrier function of the intestinal epithelium (Stoll, 2006). In piglets fed Thrdeficient diet symptoms of diarrhoea and decreased mucosal weight were recorded (Law et al., 2007).

Non-essential AA (NEAA) are also involved in the physiological functions and necessary to maintain the gut structure. Most of dietary glutamine and almost all glutamate and aspartate are intensively catabolized by the small intestinal mucosa. Only $50-70 \%$ of dietary AA enter the portal circulation. Dietary AA are major fuels for the small intestinal mucosa and are essential precursors for intestinal synthesis of proteins, glutathione, polyamines, nitric oxide etc., that play an important role in the regulation of intestinal functions (Wu et al., 2005). In our previous experiment (Święch et al., 2010), NEAA in form of wheat gluten (WG) added to low-protein diet with moderate deficiency of Thr improved gut structure in young pigs, whereas in form of monosodium glutamate had the depressive effect. Therefore, further studies on the potential interactive effects of Thr and NEAA levels on the morphology of young pigs intestine (an important component of gut health) are necessary.

The aim of the study was to determine whether and to what extent the levels of Thr and NEAA affect gut structure in young pigs fed diets balanced for all essential AA, except Thr. Wheat gluten was used as the source of NEAA, which were assumed to have sparing effect on Thr metabolism. Morphology of three segments of the small intestine (duodenum, middle jejunum and ileum) and one segment of the colon (proximal part) was evaluated in young pigs fed diets differing in standardized ileal digestibility (SID) Thr and NEAA as in the experiment reported by Święch (2015).

\section{Material and methods}

\section{Animals and experimental procedures}

The experiment was conducted on 72 castrated male Large White $\times$ Duroc pigs of $12.5 \mathrm{~kg}$ initial body weight $(\mathrm{BW})$. Animals were housed individually in cages with a slatted floor in a thermally controlled room $\left(22-23{ }^{\circ} \mathrm{C}\right)$ with free access to water. The experiment was approved by the Local Animal Care Committee (Warsaw, Poland).

Pigs were allocated to 12 groups of 6 animals according to BW, age and litter. Equal amounts of diets, after mixing with water (1:1), were given 3 times a day (8:00, 14:00 and 20:00). Pigs were fed experimental diets for approximately 20 days. The daily dietary allowance was provided at the rate of about $5 \%$ of BW. Pigs were slaughtered at about $22.4 \mathrm{~kg}$ of BW.

\section{Diets}

Diets differed in the Thr level (5.1, 5.7, 6.3 and $6.9 \mathrm{~g}$ standardized ileal digestible $\left.\mathrm{Thr} \cdot \mathrm{kg}^{-1}\right)$ and WG level $\left(20.4,40.4\right.$ and $60.4 \mathrm{~g}$ of WG protein $\cdot \mathrm{kg}^{-1}$ of respective WG20, WG40 and WG60 diets). The diets were supplemented with essential AA (excluding Thr) to cover pig AA requirements according to the ideal protein profile (Boisen, 1997). Contents of total and SID protein and AA in low-Thr diets were determined previously in digestibility experiment on cannulated pigs (Święch, 2015). WG was used as a rich source of NEAA (about $60 \%$ in WG protein) and low in Thr. All diets were formulated from the same ingredients and were free of antibiotics and other growth promoters.

\section{Sampling and histological analysis}

Immediately after slaughter, the abdominal cavity of pigs was opened and the entire gastrointestinal tract was promptly removed. The tissue samples were collected from the following gut segments: duodenum - from pyloric antrum to the point of entry of pancreatic duct, middle jejunum, ileum - the last $20 \mathrm{~cm}$ segment of the small intestine, and proximal colon. After rinsing with cold $0.9 \% \mathrm{NaCl}$ solution, $2 \mathrm{~cm}$ of tissue samples from the middle parts were taken for histological analyses.

Tissue samples were placed immediately in Bouin's solution (saturated picric acid, $40 \%$ formaldehyde and glacial acetic acid in proportion 30:15:1) for 3 days and subsequently rinsed 4 times in $70 \%$ ethanol to remove the remains of picric acid. Next, samples were dehydrated in solutions containing graded ethanol concentration (96 and 100\%) and cleaned with xylene. Tissues were infiltrated with paraffin wax. 
Table 1. Composition of experimental diets

\begin{tabular}{|c|c|c|c|}
\hline \multirow{2}{*}{ Indices } & \multicolumn{3}{|c|}{ Experimental diets ${ }^{1}$} \\
\hline & WG20 & WG40 & WG60 \\
\hline \multicolumn{4}{|l|}{ Ingredients, $\mathrm{g} \cdot \mathrm{kg}^{-1}$} \\
\hline wheat & 550.00 & 550.00 & 550.00 \\
\hline maize & 150.00 & 150.00 & 150.00 \\
\hline soyabean meal & 80.00 & 80.00 & 80.00 \\
\hline full fat soyabeans & 50.00 & 50.00 & 50.00 \\
\hline casein & 10.00 & 10.00 & 10.00 \\
\hline wheat gluten & 26.72 & 52.86 & 79.00 \\
\hline rapeseed oil & 21.00 & 18.00 & 16.00 \\
\hline calcium phosphate & 16.00 & 16.00 & 16.00 \\
\hline limestone & 12.00 & 12.00 & 12.00 \\
\hline salt & 3.50 & 3.50 & 3.50 \\
\hline vitamin-mineral mixture ${ }^{2}$ & 5.00 & 5.00 & 5.00 \\
\hline maize starch & 62.16 & 43.96 & 22.79 \\
\hline L-lysine $\mathrm{HCl}(78 \%)$ & 8.17 & 7.04 & 5.71 \\
\hline L-threonine $(98 \%)^{3}$ & 1.55 & 0.69 & \\
\hline DL-methionine (98\%) & 1.18 & 0.53 & \\
\hline L-tryptophan (99\%) & 0.37 & 0.18 & \\
\hline L-isoleucine (99\%) & 0.75 & & \\
\hline L-valine $(96.5 \%)$ & 1.38 & 0.24 & \\
\hline L-histidine (99\%) & 0.22 & & \\
\hline \multicolumn{4}{|c|}{ Content of standardized ileal digestible amino acids, $\mathrm{g} \cdot \mathrm{kg}^{-1}$} \\
\hline lysine (Lys) & 10.00 & 10.00 & 10.00 \\
\hline threonine (Thr) & 5.10 & 5.10 & 5.10 \\
\hline methionine (Met) & 2.78 & 2.78 & 2.78 \\
\hline cystine (Cys) & 2.85 & 3.24 & 3.85 \\
\hline tryptophan & 1.78 & 1.78 & 1.78 \\
\hline isoleucine & 5.50 & 6.07 & 7.67 \\
\hline valine & 6.81 & 6.80 & 8.09 \\
\hline histidine & 3.00 & 3.58 & 4.12 \\
\hline arginine & 6.11 & 7.47 & 8.93 \\
\hline leucine & 8.66 & 11.72 & 14.05 \\
\hline Thr:Lys & 0.51 & 0.51 & 0.51 \\
\hline Met:Lys & 0.28 & 0.28 & 0.28 \\
\hline Met+Cys:Lys & 0.57 & 0.60 & 0.66 \\
\hline \multicolumn{4}{|c|}{ Content of total non-essential amino acids, $\mathrm{g} \cdot \mathrm{kg}^{-1}$} \\
\hline alanine & 5.34 & 6.37 & 7.48 \\
\hline aspartic acid & 11.12 & 12.85 & 15.03 \\
\hline glutamic acid & 46.31 & 60.04 & 73.80 \\
\hline glycine & 5.52 & 6.72 & 7.90 \\
\hline proline & 12.53 & 16.74 & 19.24 \\
\hline serine & 6.85 & 8.41 & 10.25 \\
\hline tyrosine & 4.20 & 5.35 & 6.28 \\
\hline sum & 91.87 & 116.48 & 139.98 \\
\hline
\end{tabular}

${ }^{1}$ WG20, WG40, WG60 - diets containing 20.4, 40.4 and $60.4 \mathrm{~g}$ wheat gluten (WG) protein per $\mathrm{kg}$ of diet; ${ }^{2}$ provided per $\mathrm{kg}$ diet: IU: vit. $A 15000$, vit. $D_{3} 2000$; mg: vit. E 60, vit. $B_{1} 1$, vit. $B_{2} 4$, biotin 0.25 , vit. $B_{6} 3$, vit. $B_{12} 0.02$, vit. K 3 , niacin 20 , folic acid 5 , Ca panthotenian 15, choline 150, Mg 150, Mn 50, Zn 150, Se 0.3, Cu 150, Fe 125; g: Ca $1.3 ;{ }^{3}$ diets containing the lowest threonine content (5.1 g standardized ileal digestible threonine per $\mathrm{kg}$ of diet) were additionally supplemented with $0.61,1.22$, and $1.83 \mathrm{~g}$ of L-threonine to obtain diets containing $5.7,6.3$ and $6.9 \mathrm{~g}$ standardized ileal digestible threonine per $\mathrm{kg}$ of diet; L-threonine was included in diets instead of maize starch
Paraffin blocks were cut into $5 \mu \mathrm{m}$ sections using a rotary microtome (Microm HM 350, Microm GmbH; Waldorf, Germany). From each sample 2 slides containing 3 - 4 sections were prepared. The sections were deparaffinized with xylene, rehydrated through descending ethanol to water and stained with haematoxylin and counter-stained with eosin.

All measurements of the stained sections were made with a light microscope (Zeiss type Axiostar Plus, Carl Zeiss; Göttingen, Germany) at $5 \times$ and $10 \times$ magnifications using imaging analysis system (Axio Vision LE Rel 4.5 software, 2002 - 2005; Carl Zeiss; Göttingen, Germany).

For villus width at midvillus length, villus length and crypt depth measurements, 30 villi and corresponding crypts were randomly chosen from different well-oriented parts of the sections. Thickness of tunica mucosa and myenteron was also estimated. Tunica mucosa thickness was measured from the villus tip to the bottom of the crypt. The villus length was determined as the distance from the tip to the bottom of the villus and the crypt depth as the distance between the mouth of the crypt and its base. Villus surface area and villus length to crypt depth ratio were calculated.

Villus surface area (VSA) was calculated according to Law et al. (2007):

$$
\operatorname{VSA}=(\pi \cdot \mathrm{mh} \times \mathrm{h})+\pi \times\left(\frac{1}{2} \cdot \mathrm{mh}\right)^{2}
$$

where: $\mathrm{mh}$ - villus width at midvillus length; $\mathrm{h}$ - villus length measured from villus tip to villus-crypt junction.

\section{Statistical analysis}

Individual pigs were considered as the experimental unit. The results were expressed as the mean of 6 pigs. Results were subjected to two-way ANOVA procedure using SID Thr and WG levels as factors. Additionally, the effect of WG was evaluated for each SID Thr level separately using oneway ANOVA procedure. Differences among groups were evaluated using Tukey's post-hoc test. Data were statistically evaluated using Statistica 10 PL (StatSoft Inc., 2011). The effects were considered to be significant at $P \leq 0.05$.

\section{Results}

Pigs were healthy and readily consumed their daily diet allowance. Initial and final $\mathrm{BW}$ were similar in all groups (12.5 and $22.4 \mathrm{~kg}$, respectively). Growth performance of animals was uniform, mean daily body weight gain and feed : gain ratio were $550 \mathrm{~g}$ and 1.55 , respectively. 


\section{Duodenum}

The SID Thr level did not affect any of the morphological parameters, whereas WG influenced villi length, crypt depth and tunica mucosa thickness; the SID Thr · WG levels interaction was absent (Table 2). Villi were shorter $(P \leq 0.001)$ in pigs fed WG60 than WG20 and WG40 diets. Crypt depth was decreased ( $P \leq 0.001$ ) by the two higher WG levels in pigs fed diets containing 5.7 and $6.3 \mathrm{~g} \mathrm{SID} \mathrm{Thr} \cdot \mathrm{kg}^{-1}$ and tended to be decreased $(P=0.062)$ in pigs fed diet containing the lowest SID Thr level. The villi length : crypt depth ratio was higher in WG40 than WG60 group, but only at the lowest SID Thr level. Tunica mucosa was thinner $(P \leq 0.01)$ in pigs fed WG60 than WG20 and WG40 diets. Other parameters were unaffected.

\section{Middle jejunum}

Only WG affected crypt depth and myenteron thickness, however the differences were significant not for all Thr levels. The SID Thr level did not influence any of the measured parameters (Table 3). Only a slight tendency $(P=0.096)$ to the interactive effect of the two factors on myenteron thickness was found. Crypt depth was lower $(P \leq 0.05)$ in pigs fed WG60 than WG40 diet, but only with $6.3 \mathrm{~g}$ SID Thr $\cdot \mathrm{kg}^{-1}$ of diet. Myenteron was thinner $(P \leq 0.05)$ in pigs fed WG40 than WG20 and WG60 diets, however the differences were considered significant only at the lowest SID Thr level.

Table 2. Histological parameters of duodenum in pigs fed diets differing in standardized ileal digestible threonine content and wheat gluten level

\begin{tabular}{|c|c|c|c|c|c|c|c|}
\hline $\begin{array}{l}\text { Standardized ileal } \\
\text { digestible threonine, } \\
\mathrm{g} \cdot \mathrm{kg}^{-1}\end{array}$ & $\begin{array}{l}\text { Wheat gluten } \\
\text { level }^{1}\end{array}$ & $\begin{array}{l}\text { Villi length, } \\
\mu m\end{array}$ & $\begin{array}{l}\text { Villi surface } \\
\text { area, } \mathrm{mm}^{2}\end{array}$ & $\begin{array}{l}\text { Crypt depth, } \\
\mu \mathrm{m}\end{array}$ & $\begin{array}{l}\text { Villi length : crypt } \\
\text { depth ratio }\end{array}$ & $\begin{array}{l}\text { Tunica mucosa } \\
\text { thickness, } \mu \mathrm{m}\end{array}$ & $\begin{array}{l}\text { Myenteron } \\
\text { thickness, } \mu \mathrm{m}\end{array}$ \\
\hline 5.1 & 20 & 371 & 0.25 & 326 & $1.22^{\mathrm{ab}}$ & 903 & 484 \\
\hline 5.1 & 40 & 376 & 0.26 & 279 & $1.52^{\mathrm{b}}$ & 892 & 493 \\
\hline 5.1 & 60 & 336 & 0.24 & 307 & $1.20^{\mathrm{a}}$ & 860 & 489 \\
\hline \multicolumn{2}{|c|}{$P$-value (one-way ANOVA) ${ }^{2}$} & 0.219 & 0.613 & 0.062 & 0.028 & 0.483 & 0.976 \\
\hline 5.7 & 20 & $420^{\mathrm{b}}$ & 0.26 & $371^{b}$ & 1.20 & 917 & 571 \\
\hline 5.7 & 40 & $340^{\mathrm{a}}$ & 0.27 & $290^{\mathrm{a}}$ & 1.29 & 886 & 519 \\
\hline 5.7 & 60 & $332^{\mathrm{a}}$ & 0.25 & $281^{\mathrm{a}}$ & 1.34 & 834 & 563 \\
\hline \multicolumn{2}{|c|}{$P$-value (one-way ANOVA) } & 0.021 & 0.638 & 0.003 & 0.664 & 0.171 & 0.753 \\
\hline 6.3 & 20 & $430^{b}$ & 0.25 & $348^{b}$ & 1.25 & 895 & 505 \\
\hline 6.3 & 40 & $346^{\mathrm{a}}$ & 0.26 & $296^{a}$ & 1.29 & 885 & 458 \\
\hline 6.3 & 60 & $336^{a}$ & 0.24 & $290^{\mathrm{a}}$ & 1.32 & 832 & 517 \\
\hline \multicolumn{2}{|c|}{$P$-value (one-way ANOVA) } & 0.006 & 0.375 & 0.017 & 0.852 & 0.215 & 0.675 \\
\hline 6.9 & 20 & $399^{b}$ & 0.27 & 339 & 1.26 & 912 & 534 \\
\hline 6.9 & 40 & $358^{\mathrm{ab}}$ & 0.25 & 294 & 1.36 & 866 & 505 \\
\hline 6.9 & 60 & $312^{\mathrm{a}}$ & 0.25 & 297 & 1.21 & 832 & 555 \\
\hline \multicolumn{2}{|c|}{$P$-value (one-way ANOVA) } & 0.002 & 0.279 & 0.141 & 0.528 & 0.114 & 0.779 \\
\hline \multicolumn{2}{|l|}{ Pooled SEM } & 17 & 0.01 & 15 & 0.09 & 27 & 44 \\
\hline \multicolumn{8}{|c|}{ Overall means for threonine groups, $\mathrm{g} \cdot \mathrm{kg}^{-1}$} \\
\hline \multicolumn{2}{|c|}{5.1} & 361 & 0.25 & 303 & 1.32 & 885 & 489 \\
\hline \multicolumn{2}{|l|}{5.7} & 364 & 0.26 & 314 & 1.28 & 876 & 552 \\
\hline \multicolumn{2}{|l|}{6.3} & 360 & 0.25 & 310 & 1.29 & 868 & 494 \\
\hline \multicolumn{2}{|l|}{6.9} & 356 & 0.26 & 311 & 1.27 & 870 & 533 \\
\hline \multicolumn{8}{|c|}{ Overall means for wheat gluten level } \\
\hline \multicolumn{2}{|c|}{20} & $397^{b}$ & 0.26 & $345^{b}$ & 1.23 & $907^{b}$ & 521 \\
\hline \multicolumn{2}{|l|}{40} & $357^{b}$ & 0.26 & $289^{a}$ & 1.38 & $883^{b}$ & 494 \\
\hline \multicolumn{2}{|l|}{60} & $329^{a}$ & 0.24 & $295^{a}$ & 1.26 & $840^{\mathrm{a}}$ & 530 \\
\hline \multicolumn{8}{|c|}{$P$-value (two-way ANOVA) } \\
\hline \multicolumn{2}{|c|}{ standardized ileal digestible threonine } & 0.958 & 0.674 & 0.861 & 0.947 & 0.872 & 0.240 \\
\hline \multicolumn{2}{|c|}{ wheat gluten } & 0.001 & 0.176 & 0.001 & 0.137 & 0.003 & 0.452 \\
\hline \multicolumn{2}{|l|}{ interaction } & 0.252 & 0.824 & 0.399 & 0.435 & 0.981 & 0.982 \\
\hline
\end{tabular}

${ }^{1}$ see Table $1 ;{ }^{2}$ effect of wheat gluten was calculated for each standardized ileal digestible threonine level separately; ${ }^{\text {ab }}$ - means with different superscripts within a column are significantly different at $P \leq 0.05$ 
Table 3. Histological parameters of middle jejunum in pigs fed diets differing in standardized ileal digestible threonine content and wheat gluten level

\begin{tabular}{|c|c|c|c|c|c|c|c|}
\hline $\begin{array}{l}\text { Standardized ileal } \\
\text { digestible threonine, } \\
\mathrm{g} \cdot \mathrm{kg}^{-1}\end{array}$ & $\begin{array}{l}\text { Wheat gluten } \\
\text { level }^{1}\end{array}$ & $\begin{array}{l}\text { Villi length, } \\
\mu \mathrm{m}\end{array}$ & $\begin{array}{l}\text { Villi surface } \\
\text { area, } \mathrm{mm}^{2}\end{array}$ & $\begin{array}{l}\text { Crypt depth, } \\
\mu \mathrm{m}\end{array}$ & $\begin{array}{l}\text { Villi length : crypt } \\
\text { depth ratio }\end{array}$ & $\begin{array}{l}\text { Tunica mucosa } \\
\text { thickness, } \mu \mathrm{m}\end{array}$ & $\begin{array}{l}\text { Myenteron } \\
\text { thickness, } \mu \mathrm{m}\end{array}$ \\
\hline 5.1 & 20 & 436 & 0.29 & 235 & 2.03 & 779 & $385^{b}$ \\
\hline 5.1 & 40 & 421 & 0.31 & 248 & 1.85 & 775 & $314^{a}$ \\
\hline 5.1 & 60 & 417 & 0.30 & 242 & 1.88 & 778 & $394^{b}$ \\
\hline \multicolumn{2}{|c|}{$P$-value (one-way ANOVA) ${ }^{2}$} & 0.790 & 0.781 & 0.363 & 0.474 & 0.473 & 0.018 \\
\hline 5.7 & 20 & 395 & 0.28 & 244 & 1.82 & 735 & 385 \\
\hline 5.7 & 40 & 443 & 0.30 & 254 & 1.94 & 777 & 315 \\
\hline 5.7 & 60 & 407 & 0.29 & 249 & 1.80 & 784 & 387 \\
\hline \multicolumn{2}{|c|}{$P$-value (one-way ANOVA) } & 0.507 & 0.583 & 0.847 & 0.875 & 0.372 & 0.091 \\
\hline 6.3 & 20 & 443 & 0.28 & $251^{\mathrm{ab}}$ & 1.99 & 789 & 354 \\
\hline 6.3 & 40 & 378 & 0.28 & $269^{b}$ & 1.53 & 749 & 371 \\
\hline 6.3 & 60 & 409 & 0.28 & $228^{a}$ & 2.01 & 767 & 364 \\
\hline \multicolumn{2}{|c|}{$P$-value (one-way ANOVA) } & 0.330 & 0.912 & 0.033 & 0.111 & 0.411 & 0.771 \\
\hline 6.9 & 20 & 456 & 0.28 & 239 & 2.04 & 785 & 352 \\
\hline 6.9 & 40 & 426 & 0.30 & 250 & 1.85 & 767 & 373 \\
\hline 6.9 & 60 & 422 & 0.28 & 264 & 1.75 & 774 & 361 \\
\hline \multicolumn{2}{|c|}{$P$-value (one-way ANOVA) } & 0.611 & 0.654 & 0.313 & 0.323 & 0.877 & 0.792 \\
\hline \multicolumn{2}{|c|}{ Pooled SEM } & 26 & 0.01 & 10 & 0.15 & 21 & 21 \\
\hline \multicolumn{8}{|c|}{ Overall means for threonine groups, $\mathrm{g} \cdot \mathrm{kg}^{-1}$} \\
\hline \multicolumn{2}{|c|}{5.1} & 424 & 0.30 & 242 & 1.92 & 789 & 364 \\
\hline \multicolumn{2}{|l|}{5.7} & 416 & 0.29 & 249 & 1.86 & 767 & 360 \\
\hline \multicolumn{2}{|l|}{6.3} & 410 & 0.28 & 248 & 1.85 & 769 & 363 \\
\hline \multicolumn{2}{|l|}{6.9} & 436 & 0.29 & 250 & 1.89 & 781 & 363 \\
\hline \multicolumn{8}{|c|}{ Overall means for wheat gluten level } \\
\hline \multicolumn{2}{|c|}{20} & 435 & 0.28 & 241 & 1.98 & 781 & 368 \\
\hline \multicolumn{2}{|l|}{40} & 417 & 0.30 & 255 & 1.80 & 768 & 341 \\
\hline \multicolumn{2}{|l|}{60} & 414 & 0.29 & 245 & 1.87 & 779 & 373 \\
\hline \multicolumn{8}{|c|}{$P$-value (two-way ANOVA) } \\
\hline \multirow{2}{*}{\multicolumn{2}{|c|}{$\begin{array}{l}\text { standardized ileal digestible threonine } \\
\text { wheat gluten }\end{array}$}} & 0.691 & 0.451 & 0.638 & 0.909 & 0.622 & 0.999 \\
\hline & & 0.561 & 0.418 & 0.165 & 0.258 & 0.658 & 0.068 \\
\hline \multicolumn{2}{|l|}{ interaction } & 0.620 & 0.999 & 0.180 & 0.398 & 0.534 & 0.096 \\
\hline
\end{tabular}

${ }^{1}$ see Table $1 ;{ }^{2}$ effect of wheat gluten was calculated for each standardized ileal digestible threonine level separately; ${ }^{\text {ab }}$ - means with different superscripts within a column are significantly different at $P \leq 0.05$

\section{Ileum}

The SID Thr level had no effect on all analysed parameters, whereas WG affected significantly crypt depth, villi length : crypt depth ratio and myenteron thickness (Table 4). Crypt depth and myenteron thickness were higher $(P \leq 0.001)$ in pigs fed WG60 diet in comparison with WG20 diet. The effect of WG on crypt depth was significant $(P \leq 0.01)$ in pigs fed diet containing $5.7 \mathrm{~g}$ SID Thr $\cdot \mathrm{kg}^{-1}$. Villi length : crypt depth ratio was significantly higher in pigs fed WG20 diet in comparison with diet containing two higher WG levels. Wheat gluten also increased progressively tunica mucosa thickness in pigs fed the lowest SID Thr level (difference between WG60 and WG20 group at $P \leq 0.05$ ).
The interactive effects of SID Thr and WG levels were not found.

\section{Proximal colon}

The SID Thr level tended $(P=0.081)$ to affect myenteron thickness since a greater thickness was found in pigs fed diet containing $5.7 \mathrm{~g}$ SID Thr $\cdot \mathrm{kg}^{-1}$ than other SID Thr levels (Table 4). Wheat gluten depressed crypt depth when fed at the intermediate level (WG40 vs WG20; $P \leq 0.01$ ) and increased myenteron thickness when fed at the highest level (WG60 vs WG40; $P \leq 0.05$ ). Effect of WG on myenteron thickness was significant $(P \leq 0.05)$ in pigs fed diet containing $6.3 \mathrm{~g}$ SID $\mathrm{Thr} \cdot \mathrm{kg}^{-1}$. 
Table 4. Histological parameters of ileum and proximal colon in pigs fed diets differing in standardized ileal digestible threonine content and wheat gluten level

\begin{tabular}{|c|c|c|c|c|c|c|c|c|c|}
\hline \multirow[b]{2}{*}{$\begin{array}{l}\text { Standardized ileal } \\
\text { digestible threonine, } \\
\mathrm{g} \cdot \mathrm{kg}^{-1}\end{array}$} & \multirow[b]{2}{*}{$\begin{array}{l}\text { Wheat gluten } \\
\text { level }^{1}\end{array}$} & \multicolumn{6}{|c|}{ Ileum } & \multicolumn{2}{|c|}{ Proximal colon } \\
\hline & & $\begin{array}{l}\text { villi length, } \\
\mu \mathrm{m}\end{array}$ & $\begin{array}{l}\text { villi surface } \\
\text { area, } \\
\mathrm{mm}^{2}\end{array}$ & $\begin{array}{l}\text { crypt } \\
\text { depth, } \\
\mu \mathrm{m}\end{array}$ & $\begin{array}{l}\text { villi length : } \\
\text { crypt depth } \\
\text { ratio }\end{array}$ & $\begin{array}{l}\text { tunica mucosa } \\
\text { thickness, } \\
\mu \mathrm{m}\end{array}$ & $\begin{array}{l}\text { myenteron } \\
\text { thickness, } \\
\mu \mathrm{m}\end{array}$ & $\begin{array}{l}\text { crypt } \\
\text { depth, } \\
\mu \mathrm{m}\end{array}$ & $\begin{array}{l}\text { myenteron } \\
\text { thickness, } \\
\mu \mathrm{m}\end{array}$ \\
\hline 5.1 & 20 & 320 & 0.15 & 216 & 1.60 & $541^{\mathrm{a}}$ & 761 & 420 & 368 \\
\hline 5.1 & 40 & 311 & 0.15 & 229 & 1.50 & $556^{\mathrm{ab}}$ & 780 & 398 & 380 \\
\hline 5.1 & 60 & 343 & 0.17 & 239 & 1.55 & $598^{b}$ & 859 & 401 & 383 \\
\hline \multicolumn{2}{|c|}{$P$-value (one-way ANOVA) ${ }^{2}$} & 0.089 & 0.077 & 0.160 & 0.683 & 0.049 & 0.443 & 0.300 & 0.915 \\
\hline 5.7 & 20 & 327 & 0.17 & $215^{\mathrm{a}}$ & 1.63 & 554 & 774 & 414 & 471 \\
\hline 5.7 & 40 & 303 & 0.18 & $220^{\mathrm{a}}$ & 1.51 & 543 & 791 & 373 & 393 \\
\hline 5.7 & 60 & 314 & 0.18 & $245^{b}$ & 1.37 & 574 & 840 & 386 & 478 \\
\hline \multicolumn{2}{|c|}{$P$-value (one-way ANOVA) } & 0.607 & 0.916 & 0.007 & 0.087 & 0.599 & 0.720 & 0.112 & 0.642 \\
\hline 6.3 & 20 & 325 & 0.17 & 212 & 1.65 & 545 & 703 & 412 & $366^{\mathrm{ab}}$ \\
\hline 6.3 & 40 & 307 & 0.15 & 226 & 1.47 & 554 & 758 & 379 & $302^{a}$ \\
\hline 6.3 & 60 & 305 & 0.16 & 242 & 1.37 & 563 & 865 & 404 & $440^{b}$ \\
\hline \multicolumn{2}{|c|}{$P$-value (one-way ANOVA) } & 0.608 & 0.177 & 0.145 & 0.087 & 0.862 & 0.072 & 0.196 & 0.020 \\
\hline 6.9 & 20 & 324 & 0.16 & 205 & 1.70 & 547 & 754 & 396 & 348 \\
\hline 6.9 & 40 & 306 & 0.16 & 223 & 1.50 & 550 & 758 & 368 & 297 \\
\hline 6.9 & 60 & 304 & 0.17 & 226 & 1.47 & 534 & 892 & 395 & 444 \\
\hline \multicolumn{2}{|c|}{$P$-value (one-way ANOVA) } & 0.466 & 0.788 & 0.361 & 0.155 & 0.865 & 0.230 & 0.272 & 0.055 \\
\hline \multicolumn{2}{|c|}{ Pooled SEM } & 13 & 0.01 & 9 & 0.08 & 21 & 57 & 12 & 43 \\
\hline \multicolumn{10}{|c|}{ Overall means for threonine groups, $\mathrm{g} \cdot \mathrm{kg}^{-1}$} \\
\hline \multicolumn{2}{|c|}{5.1} & 325 & 0.16 & 228 & 1.55 & 565 & 800 & 406 & 377 \\
\hline \multicolumn{2}{|l|}{5.7} & 315 & 0.17 & 228 & 1.50 & 558 & 804 & 392 & 449 \\
\hline \multicolumn{2}{|l|}{6.3} & 312 & 0.16 & 228 & 1.49 & 555 & 780 & 399 & 373 \\
\hline \multicolumn{2}{|l|}{6.9} & 312 & 0.17 & 217 & 1.56 & 543 & 803 & 387 & 366 \\
\hline \multicolumn{10}{|c|}{ Overall means for wheat gluten level } \\
\hline \multicolumn{2}{|c|}{20} & 324 & 0.16 & $212^{\mathrm{a}}$ & $1.64^{\mathrm{b}}$ & 546 & $749^{a}$ & $410^{\mathrm{b}}$ & $385^{\mathrm{ab}}$ \\
\hline \multicolumn{2}{|l|}{40} & 307 & 0.16 & $225^{\mathrm{ab}}$ & $1.50^{\mathrm{a}}$ & 551 & $772^{\mathrm{ab}}$ & $382^{\mathrm{a}}$ & $346^{a}$ \\
\hline \multicolumn{2}{|l|}{60} & 317 & 0.16 & $238^{b}$ & $1.45^{\mathrm{a}}$ & 568 & $863^{b}$ & $396^{\mathrm{ab}}$ & $436^{b}$ \\
\hline \multicolumn{10}{|c|}{$P$-value (two-way ANOVA) } \\
\hline \multicolumn{2}{|c|}{ standardized ileal digestible threonine } & 0.564 & 0.352 & 0.478 & 0.747 & 0.634 & 0.933 & 0.193 & 0.081 \\
\hline \multicolumn{2}{|c|}{ wheat gluten } & 0.206 & 0.395 & 0.001 & 0.002 & 0.328 & 0.013 & 0.003 & 0.012 \\
\hline \multicolumn{2}{|l|}{ interaction } & 0.618 & 0.792 & 0.952 & 0.775 & 0.673 & 0.983 & 0.877 & 0.613 \\
\hline
\end{tabular}

${ }^{1}$ see Table $1 ;{ }^{2}$ effect of wheat gluten was calculated for each standardized ileal digestible threonine level separately; ${ }^{\text {ab }}$ - means with different superscripts within a column are significantly different at $P \leq 0.05$

\section{Discussion}

The present study did not show any effect of Thr level on the intestinal structure of growing pigs and this result is apparently contradictory to the wellestablished data on the beneficial influence of this AA in the gut. The positive influence of Thr supplementation on villi length and tunica mucosa was found in our previous studies (Święch et al., 2011) on pigs of similar BW fed Thr-deficient diet ( $87 \%$ requirement), and on morphology of all segments of the small intestine in older pigs fed diet covering $80 \%$ of $\mathrm{Thr}$ requirement (Święch et al., 2010). Feeding diet with greater $\mathrm{Thr}$ deficiency (70\% requirement) reduced villi length and villi length: crypt depth ratio in the ileum of early weaned piglets (Hamard et al., 2007), whereas diet covering only $17 \%$ of requirement decreased these parameters in the middle jejunum and ileum (Law et al., 2007). Also in studies of Wang et al. (2010) and Ren et al. (2014), feeding diets covering $50 \%$ of $\mathrm{Thr}$ requirement resulted in decreasing villus length in the duodenum and ileum, and in lowering villi length : crypt depth ratio in the duodenum and jejunum, respectively. Deformations of the intestinal morphology were observed not only due to the deficiency, but also to the excess of $\operatorname{Thr}(150 \%$ of requirement) (Wang et al., 2010; Ren et al., 2014). 
The possible explanation of the discrepancy between results of the present and reported studies may be caused by too small deficiency in diet with the lowest level of SID Thr $\left(5.1 \mathrm{~g}\right.$ SID Thr $\cdot \mathrm{kg}^{-1}$ of diet) assumed as deficient. This explanation is supported by the absence of any effect of Thr on the nitrogen retention determined in the same piglets (Święch, 2015). The Thr requirement, that can be found in literature, is not strictly uniform and the actual requirement may also vary to some extent depending on dietary factors, such as protein and lysine levels, and on pig performance.

The intestinal structure in pigs fed presumably Thr-deficient diet did not show any anomalies and was not improved by additional Thr supplementation. It should be underlined that, in spite of the absence of any effect of Thr in the small intestine, the increase of Thr level from 5.1 to $5.7 \mathrm{~g} \mathrm{SID} \mathrm{Thr} \cdot \mathrm{kg}^{-1}$ (but not more) tended to increase myenteron thickness in the proximal colon. This distant effect of Thr should be confirmed, but seems warrantable.

The effect of supplementing diets with increasing amounts of WG on morphological parameters was predominantly negative and differed among the segments. Villi length was affected by WG only in the duodenum, where WG level had no effect at the lowest Thr level, but it reduced villi length at three higher Thr contents. It should be, however, underlined that across all higher Thr levels, villi length recorded in pigs fed WG20 diet was particularly high. Crypt depth in the duodenum was also not affected by WG at the lowest Thr level and in pigs fed diet with greater amounts of Thr, it was the greatest in pigs fed WG20 diets. Crypt depth was affected by WG level in the ileum, where the increase of WG supplement increased or tended to increase crypt depth, which may indicate less mature enterocytes on the ileal villi and reduced capacity for digestion and absorption. These results are partly in agreement with the varied effect of 20 and $40 \mathrm{~g}$ of WG protein and depressive effect of monosodium glutamate on villus length and crypt depth in different parts of the small intestine found in our previous study (Święch et al., 2010). However, in Kitt et al. (2003) study the supplementation of comparatively small amounts of NEAA ( $5 \%$ glutamine or mixture of glycine, alanine, asparagine, proline and serine) to the diet did not influence villi length in the duodenum and jejunum.

The villous length : crypt depth ratio has been proposed as the criterion of digestive capacity of the small intestine (Montagne et al., 2003). In the present study, this ratio in the ileum was highest in pigs fed WG20 diets and was decreased by both WG levels due to the deeper crypts without a change in villous length. In contrast, changes of both parameters caused by WG in the duodenum did not provoke significant modification of their ratio.

The reduction of villi length and crypt depth in the duodenum by increasing WG level cannot be attributed to lower feed intake since in all groups it was maintained at the similar level (Święch, 2015). Neither it can be ascribed to the increase of dietary protein content from 17 to 19 and $21 \%$ in WG20, WG40 and WG60 diets, respectively, since the opposite stimulating effects of the protein level on these parameters were found (Gu and Li, 2004; Nyachoti et al., 2006). The total content of NEAA in WG40 and WG60 diets was greater by 26 and $52 \%$ than in WG20 diet and glutamic acid contributed for over $50 \%$ of the NEAA. It may be therefore assumed that these AA are actually responsible for the deterioration of the epithelial structure of the duodenum.

The morphology of villi and crypt is considered as related to the digestive and absorptive capacity of the intestine. Villus atrophy and crypt hyperplasia often found in weaned piglets (Pluske et al., 1997) are associated with dramatic depression of small intestine digestive functions. In our study, the recorded changes in these two parameters due to increased WG level were not sufficient to provoke reduction of ileal digestibility of protein and AA, as found in the previous experiment by Święch (2015).

In the present study, tunica mucosa was influenced differently by WG level in the duodenum and ileum. In the duodenum, it was the thickest in pigs fed diet with the two lower WG levels, whereas in the ileum - in pigs fed the highest WG level. These results are in line with the effects of WG on crypt depth in these two segments. The effects of WG on tunica mucosa are partially in agreement with our previous findings that mucosa thickness was greater in the duodenum in pigs fed Thr-deficient diet supplemented with $40 \mathrm{~g}$ WG protein, while it was decreased by monosodium glutamate in all small intestine segments (Święch et al., 2010).

The effects of WG level on myenteron thickness differed among the segments. They were absent in the duodenum, while in the middle jejunum tended to be Thr content depended ( $P=0.096$ for interaction). At the two lower Thr levels myenteron was the thinnest in pigs fed diet supplemented with WG40, whereas in pigs fed diet with higher Thr content myenteron thickness was not affected by WG. In the ileum and proximal colon, WG60 diet 
considerably increased the myenteron thickness. The greater myenteron thickness in these gut segments may be associated with a faster passage of digesta and, in consequence, with the rate of processes in the hind gut. The results of the present experiment are in agreement with the absence of WG effect on myenteron thickness in the duodenum and middle jejunum and decreased myenteron thickness in the ileum reported by Święch et al. (2010). These authors found the opposite effect of monosodium glutamate, which decreased myenteron thickness in the duodenum and did not influence it in the middle jejunum and ileum.

The depressive and irregular effects of increasing amounts of NEAA added in form of WG on gut morphology are not in agreement with our previous results and are difficult to explain. Deterioration of some parameters of gut morphology was, however, not great enough to depress the digestive functions of the small intestine estimated with the same diets and pigs.

\section{Conclusions}

The absence of the expected effects of increased standardized ileal digestible threonine level on gut morphology may be ascribed to a satisfactory supply of threonine, even at the lowest level, and is in line with absence of threonine effect on nitrogen retention found in the same animals. Lack of the sparing effect of non-essential amino acids added in form of wheat gluten does not confirm the opinion on their generally positive effects on the parameters involved in the digestive functions.

\section{Acknowledgements}

The research was supported by The Polish National Science Centre, Project No. N N311 316736.

\section{References}

Bertolo R.F.P., Chen C.Z.L., Law G., Pencharz P.B., Ball R.O., 1998. Threonine requirement of neonatal piglets receiving total parenteral nutrition is considerably lower than that of piglets receiving an identical diet intragastrically. J. Nutr. 128, 1752-1759

Boisen S., 1997. Ideal protein - and its suitability to characterize protein quality in pig feeds. A review. Acta Agr. Scand. Sect. A - Anim. Sci. 47, 31-38

Burrin D.G., Stoll B., van Goudoever J.B., Reeds P.J., 2001. Nutrient requirements for intestinal growth and metabolism in the developing pigs. In: Proceedings of the $8^{\text {th }}$ Symposium on Digestive Physiology of Pigs. Uppsala (Sweden), pp. 75-88
Cera K.R., Maham D.C., Cross R.F., Reinhart G.A., Whitmoyer R.E., 1988. Effect of age, weaning and postweaning diet on small intestinal growth and jejunal morphology in young swine. J. Anim. Sci. 66, 574-584

Dunsford B.R., Knabe D.A., Haensly W.E., 1989. Effect of dietary soybean meal on the microscopic anatomy of the small intestine in the early-weaned pig. J. Anim. Sci. 67, 1855-1863

Gu X., Li D., 2004. Effect of dietary crude protein level on villous morphology, immune status and histochemistry parameters of digestive tract in weaning piglets. Anim. Feed Sci. Tech. 114, 113-226

Hamard A., Sève B., Le Floc'h N., 2007. Intestinal development and growth performance of early-weaned piglets fed a low-threonine diet. Animal 1, 1134-1142

Kitt S.J., Miller P.S., Fischer R.L., 2003. Effects of dietary glutamine on growth performance and small intestine weight and length of weanling pigs before and after an immune challenge. In: Proceedings of $9^{\text {th }}$ Symposium on Digestive Physiology in Pigs. Banff (Canada), Vol. 2, pp. 337-339

Law G.K., Bertolo R.F., Adjiri-Awere A., Pencharz P.B., Ball R.O., 2007. Adequate oral threonine is critical for mucin production and gut function in neonatal piglets. Am. J. Physiol. - Gastrointest. Liver Physiol. 292, G1293-G1301

Montagne L., Pluske J.R., Hampson D.J., 2003. A review of interactions between dietary fibre and the intestinal mucosa, and their consequences on digestive health in young non-ruminant animals. Anim. Feed Sci. Tech. 108, 95-117

Nyachoti C.M., Omogbenigun F.O., Rademacher M., Blank G., 2006. Performance responses and indicators of gastrointestinal health in early-weaned pigs fed low-protein amino acid-supplemented diets. J. Anim. Sci. 84, 125-134

Pluske J.R., Hampson D.J., Williams I.H., 1997. Factors influencing the structure and function of the small intestine in the weaned pig: a review. Livest. Prod. Sci. 51, 215-236

Ren M., Liu X.T., Wang X., Zhang G.J., Qiao S.Y., Zeng X.F., 2014. Increased levels of standardized ileal digestible threonine attenuate intestinal damage and immune responses in Escherichia coli $\mathrm{K}^{+} 8^{+}$challenged weaned piglets. Anim. Feed Sci. Tech. 195, 67-75

StatSoft Inc., 2011. STATISTICA (data analysis software) Version 10, www.statsoft.com

Stoll B., 2006. Intestinal uptake and metabolism of threonine: nutritional impact. Adv. Pork Prod. 17, 257-263

Stoll B., Henry J., Reeds P.J., Yu H., Jahoor F., Burrin D.G., 1998. Catabolism dominates the first-pass intestinal metabolism of dietary essential amino acids in milk protein-fed piglets. J. Nutr. 128, 606-614

Święch E., 2015. Utilization of threonine in young pigs fed diets differing in standardized ileal digestible threonine and wheat gluten used as a source of non-essential amino acids. J. Anim. Feed Sci. 24, 323-331

Święch E., Boryczka M., Taciak M., Buraczewska L., 2011. The effect of graded levels of dietary threonine on nitrogen retention and structure of the small intestine in young pigs. J. Anim. Feed Sci. 20, 350-360

Święch E., Buraczewska L., Tuśnio A., Taciak M., 2010. The effects of supplementing a low-protein threonine-deficient diet with different sources of non-essential amino acids on nitrogen retention and gut structure in young pigs. Arch. Anim. Nutr. 64, 22-35

Wang W., Zeng X., Mao X., Wu G., Qiao S., 2010. Optimal dietary true ileal digestible threonine for supporting the mucosal barrier in small intestine of weaning pigs. J. Nutr. 140, 981-986 
Wang X., Qiao S.Y., Liu M., Ma Y.X., 2006. Effects of graded levels of true ileal digestible threonine on performance, serum parameters and immune function of 10-25 kg pigs. Anim. Feed Sci. Tech. 129, 264-278

Wang X., Qiao S., Yin Y., Yue L., Wang Z., Wu G., 2007. A deficiency or excess of dietary threonine reduces protein synthesis in jejunum and skeletal muscle of young pigs. J. Nutr. 137, 1442-1446
Wu G., Knabe D.A., Flynn N.E., 2005. Amino acid metabolism in the small intestine: biochemical bases and nutritional significance. In: D.G. Burrin, H.J. Mersmann (Editors). Biology of Metabolism in Growing Animals. Elsevier, Amsterdam (The Netherlands), pp. 107-126 